\title{
LA LEGITIMACIÓN PASIVA DE LOS HOSPITALES AUTOGESTIONADOS*
}

\author{
THE LEGAL STANDING TO BE SUED OF SELF-MANAGED HOSPITALS \\ LA LEGITIMATION PASIVES DES HÔPITAUX AUTOGÉRÉS
}

\author{
Carolina Carvajal Tadres ${ }^{* *}$
}

\begin{abstract}
RESUMEN
En este trabajo se examina para el caso particular de la responsabilidad sanitaria estatal, la legitimación pasiva de los Hospitales Autogestionados. Mayoritariamente, la doctrina y jurisprudencia han sostenido que las acciones indemnizatorias derivadas de daños ocasionados por Hospitales Autogestionados por falta de servicio, deben dirigirse directamente en contra de los Servicios de Salud de quienes dependen, sin embargo, esta noción se ve cuestionada al considerar que esta clase de hospitales no responde a estructuras organizacionales administrativas clásicas y no sigue los criterios tradicionales de atribución de capacidad para ser parte de los entes públicos.
\end{abstract}

Palabras CLAVE: Hospital - Servicios de salud - Autogestión - Legitimación pasiva

ABSTRACT

This essay studies the situation of the government healthcare liability, specifically if in this case a self-managed Hospital has legal standing to be sued. Most doctrine and precedent have considered that the torts lawsuits for damages caused by Self-Managed Hospitals due to lack of service, should be directed against the Local Health Service theyre dependent of. However, this notion is challenged herein, considering that this type of hospitals don't respond to conventional administrative organizational structures and don't follow the traditional criteria for attributing legal capacity to be part of public entities.

KEYWORDS: Hospital - Local health services - Self-management - legal standing to be sued RÉSUMÉ

A propos de la responsabilité sanitaire de l'Etat, la legitimation passive des hôpitaux auto gérés est l'objet de cette chronique. La plupart de la Doctrine et la jurisprudence ont soutenu que les actions de dommages-intèrêts par dommage cause par hôpitaux auto gérés par faute de service doit se diriger contre les Services de la Santé; cependant, cette notion doi têtre mise en question selon le type d'hôpitaux auto gérés, car ces hôpitaux ne répondent point à une structure administrative classique et on ne peut pas l'attribuer les critères traditionaux d'attribution de capacité pour être partie des organs publics.

MOTS CLÉS: Hôpital - Services de santé - Autogestion - Legitimation passive

\footnotetext{
* Artículo recibido el 10 de septiembre de 2014 y aceptado para su publicación el 15 de noviembre de 2014.

** Abogado. Licenciada en Ciencias Jurídicas y Sociales por la Universidad Diego Portales. Alumna del programa de Magíster en Derecho con o sin Mención de la Universidad de Chile. Jefe Unidad de Asesoría Jurídica del Instituto Psiquiátrico “Dr. José Horwitz Barak”, Santiago, Chile. Correo electrónico: carolina. carvajal@redsalud.gov.cl.
}

Revista de Derecho · Escuela de Postgrado No 6, diciembre 2014

Páginas $137-158$

ISSN 0719 - 1731 


\section{INTRODUCCIÓN}

La más reciente literatura jurídica, en materia de responsabilidad estatal por daños sanitarios, al referirse a la legitimación pasiva de los servicios públicos de salud, sostiene que las acciones indemnizatorias derivadas de daños ocasionados por falta de servicio por los Hospitales Autogestionados deben dirigirse directamente en contra de los Servicios de Salud, de quienes dependen jurídica y administrativamente, quienes responderían patrimonialmente por éstos ${ }^{1}$.

Esta afirmación no considera que la Reforma a la Salud, específicamente a través de la Ley No 19.937, sobre Autoridad Sanitaria y Gestión², instauró una nueva regulación dirigida a mejorar la eficiencia y la calidad de las provisiones públicas de salud. Entre otras transformaciones, asignó un nuevo rol de "Gestores de Red" a los Servicios de Salud y creó la categoría de Hospitales Autogestionados en Red como entes funcionalmente desconcentrados de estos, otorgándoles facultades que implican una mayor autonomía organizacional, administrativa y presupuestaria; y generándoles, a modo de contrapartida de estas mayores atribuciones, una responsabilidad patrimonial directa ${ }^{3}$. Esto se traduce en la práctica en que, a diferencia de lo que sostiene la doctrina, son los propios Hospitales Autogestionados y no

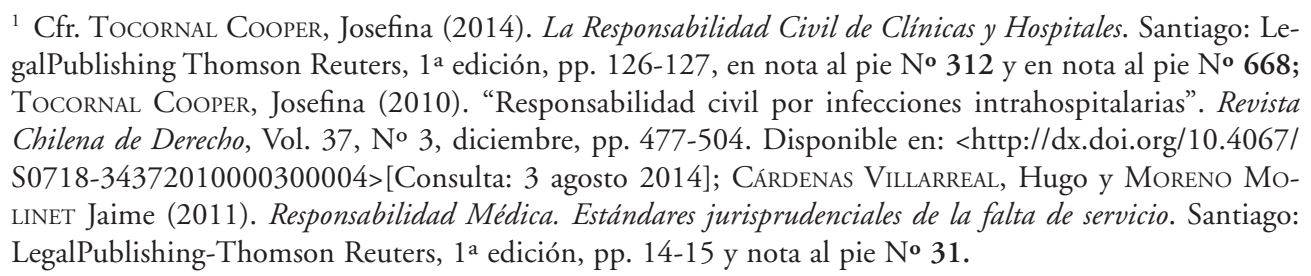
${ }^{2}$ Ley No 19.937, modifica el D.L. No 2.763 de 1979 con la finalidad de establecer una nueva concepción de la autoridad sanitaria, distintas modalidades de gestión y fortalecer la participación ciudadana, Diario Oficial, 24 de febrero de 2004.

${ }_{3}$ Para revisar una útil organización de contenidos sobre Hospitales Autogestionados, véase: NANCUANTE Almonacid, Ulises y Romero Celedón, Andrés (2008). La reforma de la salud. Instituto de Políticas Públicas y Gestión, Salud y Futuro-UNAB, Santiago: Editorial Biblioteca Americana, 1 a edición, pp. 359-392; Nancuante Almonacid, Ulises, Romero Celedón, Andrés, y Sotomayor Klapp, Roberto (2012). Régimen jurídico de la salud. Santiago: LegalPublishing Thomson Reuters, $1^{a}$ edición, pp.160-188; INOSTROZA, Manuel y NanCUANTE, Ulises (2013). "La experiencia chilena de administrar los Hospitales Autogestionados en Red 2005-2012: ¿Un instrumento consolidado y definitivo o una transición a un nuevo tipo de prestador público, para una nueva etapa de la Reforma de Salud". 95 propuestas para un Chile mejor, Estudio de Trasfondo Grupo Res Publica Chile, Capítulo 15, pp. 121-181. Disponible en: <http://95propuestas.cl/site/ wp-content/uploads/2013/05/la-experiencia-chilena-de-administrar-los-hospitales-autogestionados-en-redmanuel-inostroza-y-ulises-nancuante.pdf>[Consulta: 3 agosto 2014]. También sobre Hospitales Autogestionados: Méndez, Claudio A., Miranda, Christian, Torres, M. Cristina, y Márquez, Myriam (2012). "Implementación de la política de hospitales autogestionados en Chile: percepción de los profesionales hospitalarios". Revista Chilena de Salud Pública, Vol. 16, No 3, pp. 247-255. Disponible en: <http://www. revistasaludpublica.uchile.cl/index.php/RCSP/article/download/23158/24509> [consulta: 03 agosto 2014]; Méndez, Claudio A., Miranda, Christian, Torres, M. Cristina, y Márquez, Myriam (2013), "Política de autogestión hospitalaria en Chile: percepciones de los tomadores de decisiones". Revista Panameña de Salud Pública, Vol. 33, No 1, pp. 47-53. Disponible en: <http://www.captura.uchile.cl/handle/2250/133406> [Consulta: 3 agosto 2014]. 
los Servicios de Salud, quienes detentan la legitimación pasiva en las acciones de responsabilidad por falta de servicio y la titularidad jurídica para responder patrimonialmente por las indemnizaciones compensatorias de perjuicios.

Este modelo de gestión hospitalaria, como se verá, es complejo y el problema de entender la legitimación pasiva de los Hospitales Autogestionados debe abordarse por una parte, considerándolos como una institución que no responde a estructuras organizacionales administrativas clásicas, y por otra, reparando en que la doctrina, para explicar este tema, ha recurrido a una jurisprudencia desactualizada en relación al proceso de Reforma de la Salud.

En el presente trabajo, trataré la legitimación pasiva de los Hospitales Autogestionados partiendo con una breve reseña de la organización administrativa de los Servicios de Salud y de los Hospitales Autogestionados y las características de la desconcentración administrativa de estos últimos (1), para luego presentar el tema de la legitimación pasiva desde los criterios tradicionales de atribución de capacidad para ser parte y mediante el análisis de tres sentencias referidas a la responsabilidad sanitaria de los hospitales y servicios públicos de salud: "Astete Ortiz cl Hospital Luis Calvo Mackenna (2009)4, Sánchez Candia cl Hospital Luis Calvo Mackenna (2010)5, y“Contreras Vásquez cl Hospital Barros Luco Trudeau” (2014)6 (2).

\section{LA ORGANIZACIÓN ADMINISTRATIVA DE LOS SERVICIOS DE SALUD Y DE LOS HOSPITALES AUTOGESTIONADOS}

Desde mediados de los cincuenta y hasta los años ochenta ${ }^{7}$, el sistema público de salud chileno no era único sino que se encontraba segmentado por categoría laboral, según si se trataba de la atención de obreros o de empleados ${ }^{8}$. Estaba, por

\footnotetext{
4 "Astete Ortiz cl Hospital Luis Calvo Mackenna" (indemnización de perjuicios): 27o Juzgado Civil de Santiago, 13 de marzo de 2006, Rol No 1733-2000; Corte Apelaciones de Santiago, 7 de agosto de 2009, Rol No 3648-2006; Corte Suprema, 9 de diciembre de 2011, Rol No 7623-2009. (cit. Cárdenas Villarreal y Moreno Molinet (2011), pp. 14-15 en nota al pie No 33; Tocornal Cooper (2014), p. 1236 en nota al pie No 312).

5 "Sánchez Candia cl Hospital Luis Calvo Mackenna" (indemnización de perjuicios): 120 Juzgado Civil de Santiago, 29 de abril de 2009, Rol No 16763-2006; Corte Apelaciones de Santiago, 29 de mayo de 2010, Rol No 4046-2009 (cit. Cárdenas Villarreal y Moreno Molinet (2011), pp. 14-15 en nota al pie No 33).

6 "Contreras Vásquez cl Hospital Barros Luco Trudeau" (indemnización de perjuicios): 2o Juzgado Civil de San Miguel, 30 de octubre de 2013, Rol No 75882-2011; Corte Apelaciones de San Miguel, 22 de enero de 2014, Rol No 2292-2013.

7 Sobre la historia de la salud en Chile desde 1552 a la actualidad, Véase: Ministerio de SAlud. "Hitos de la salud chilena”.Disponible en: <http://web.minsal.cl/hitos_salud_chilena>[Consulta: 3 agosto 2014]; e "Historia del MINSAL". Disponible en: <http://web.minsal.cl/historia_minsal>[Consulta: 3 agosto 2014]; Colegio Médico de Chile. "El Sistema de Salud Chileno: contexto histórico y normativo del sistema de salud chileno". Disponible en: <http://www.colegiomedico.cl/Portals/0/files/biblioteca/documentos/otros/ Sistemasaludchileno.pdf> [Consulta: 3 agosto 2014].

${ }^{8}$ La distinción entre empleados y obreros, basada en la preeminencia del esfuerzo intelectual o del esfuerzo físico, existió en la legislación para efectos laborales hasta el año 1978, en que fue definitivamente suprimida
} 
un lado, el Servicio Nacional de Salud (SNS) ${ }^{9}$ que atendía las prestaciones de los obreros e indigentes, y por el otro, se encontraba el Servicio Médico Nacional de Empleados (SERMENA), que financiaba las prestaciones de salud de los empleados activos o pasivos afiliados a las Cajas de Previsión de Empleados Públicos y Particulares, las cuales se desarrollaban bajo una modalidad institucional en los establecimientos del SNS o de libre elección a través de proveedores privados ${ }^{10}$. Ambos Servicios se articulaban en torno al Ministerio de Salud (en ese entonces denominado Ministerio de Salubridad, Previsión y Asistencia Social ${ }^{11}$.

El Servicio Nacional de Salud (SNS), creado por la Ley No 10.383, de 1952, se instituyó como el servicio público encargado de la protección de la salud de toda la población por medio de la ejecución directa de las acciones de salud (artículo 62 Ley No 10.383). Si bien administrativamente se organizó como un servicio público con personalidad jurídica y patrimonio propio, dependiente del Ministerio de Salud (artículos 65 y 67 Ley No 10.383) y organizado territorialmente en zonas de salud (artículo 74 Ley No 10.383) dispuestas en torno a un hospital base con consultorios periféricos ligados a él; su gestión organizacional tuvo una orientación predominante de planificación central, puesto que, en definitiva, ejerció las funciones de administrar y operar todo el sistema público de salud ${ }^{12}$. Ello significó, en la práctica,

por el D.L. No 2.200. Sin embargo, para efectos previsionales, dicho decreto ley mantuvo esa diferencia, la que aún subsiste, encontrándose actualmente consagrada en el artículo $1^{\circ}$ transitorio del Código del Trabajo y en el artículo 83 del D.L. No 3.500 de 1980. Actualmente esta discriminación existe en la Ley No 16.744, que establece normas sobre accidentes del trabajo y enfermedades profesionales, Diario Oficial, 1 de febrero de 1968.

${ }^{9}$ El SNS creado por Ley No 10.383, que modifica ley 4.054 relacionada con el seguro obligatorio, Diario Oficial, 8 de agosto de 1952, como resultado de la fusión de diversas instituciones que otorgaban atención sanitaria. Conforme establece el artículo 63 de la Ley No 10.383, las instituciones fusionadas fueron el Servicio Médico de la Caja de Seguro Obrero (1924), el Servicio Nacional de Salubridad (1925), la Junta Central de Beneficencia y Asistencia Social (1832), la Dirección General de Protección a la Infancia y Adolescencia (PROTINFA, 1942); la sección técnica de Higiene y Seguridad Industrial de la Dirección General del Trabajo; los servicios médicos y sanitarios de las municipalidades; y el Instituto Bacteriológico de Chile (1929). Asimismo, según el artículo 64, se facultó al Presidente de la República para disponer, cuando las circunstancias lo aconsejaran, la incorporación al Servicio Nacional de Salud de otros servicios fiscales, semifiscales o municipales de carácter médico y asistenciales.

${ }^{10}$ El SERMENA fue creado en 1942 por el D.F.L. No 32/1552, constituido por la unión de diferentes departamentos de salud del conjunto de Cajas de Previsión de Empleados Públicos y Particulares. La modalidad de libre elección operó a contar de la Ley No 16.781 de medicina curativa del año 1968.

11 Vergara, Marcos (2007). "Reforma del sector de la salud en Chile: Avances y problemas en la implementación”. Serie En Foco No 112. Disponible en: <http://www.expansiva.cl/publicaciones/en_foco/detalle. tpl?iddocumento $=04072007114141>$ [Consulta: 3 agosto 2014].

${ }^{12}$ Goic G., Alejandro y Armas M., Rodolfo (2003). “Descentralización en salud y educación: La experiencia chilena”. Revista Médica de Chile, Vol. 131, No 7, pp. 788-798. Disponible en: <http://dx.doi.org/10.4067/ S0034-98872003000700012> [Consulta: 3 agosto 2014]. En el mismo sentido, Titelman, Daniel (2000). "Reformas al Sistema de Salud en Chile: Desafíos pendientes". CEPAL-Serie Financiamiento del Desarrollo, Unidad de Financiamiento para el Desarrollo, División de Comercio Internacional y Financiamiento para el Desarrollo, Naciones Unidas,Vol. 104, septiembre. Disponible en: <www.cepal.org/publicaciones/ xml/0/5640/lcl1425e.pdf> [Consulta: 3 agosto 2014]. 
que la Administración central asumiera la responsabilidad de generar la oferta y satisfacer las necesidades de salud pública y, consecuentemente, se atribuyera todas las potestades y funciones políticas, económicas y organizacionales necesarias para ello. Esto no impedía, evidentemente, la existencia de agentes públicos territoriales en todo el Estado, como Hospitales o Consultorios, pero estos se encontraban sujetos a la autoridad del SNS por vínculos de jerarquía, pasando a tener el carácter de simples circunscripciones de una misma administración estatal ${ }^{13}$.

En el año 1979, mediante el Decreto Ley No 2.763, se descentralizó administrativa y financieramente el sistema público de salud ${ }^{14}$. Se fusionaron los antiguos Servicio Nacional de Salud (SNS) y SERMENA, originando el Sistema Nacional de Servicios de Salud (SNSS), compuesto por Servicios de Salud a cargo de la ejecución directa de acciones de salud y de facultades fiscalizadoras en materia sanitaria (artículo 16 D.L. No 2.763/1979) ${ }^{15}$. Los Servicios de Salud se organizaron administrativamente como servicios públicos funcionalmente descentralizados, con personalidad jurídica y patrimonio propio, sujetos a la supervigilancia del Ministerio de Salud, cuyas políticas, normas y planes generales debían ejecutar (artículo 16 incisos $2^{\circ}$ y $3^{\circ}$ D.L. No 2.763/1979). El Ministerio de Salud, por su parte, pasó a cumplir un rol normativo, de supervisión y de control (artículo $4^{\circ}$ D.L. No 2.763/1979).

Que los Servicios de Salud se hayan organizado como entes descentralizados funcionalmente significó, en los términos del actual artículo 29 inciso $2^{\circ}$ de la Ley No 18.575, Orgánica Constitucional de Bases Generales de la Administración del Estado (en adelante $\mathrm{LOCBGAE}^{16}$, transferirles desde la Administración central, la titula-

\footnotetext{
13 Ferrada Bórquez, Juan Carlos (1999). "El Estado Administrador en Chile: de unitario centralizado a descentralizado y desconcentrado". Revista de Derecho (Valdivia), Vol. X, diciembre, pp. 115-123. Disponible en: <http://mingaonline.uach.cl/scielo.php?pid=S0718-09501999000200011\&script=sci_arttext> [Consulta: 3 agosto 2014].

${ }^{14}$ El proceso de descentralización de la salud continuó en el año 1980 cuando mediante los D.F.L. No 1-3063, D.L. No 3.477 y D.L. No 3.529, se traspasaron los Consultorios de Atención Primaria a la administración municipal. Sobre la restructuración y reformas del sistema de salud estatal chileno implementadas en la década de los años 80 , con especial énfasis en los procesos de descentralización y privatización, véase Miranda R., Ernesto (1990). "Descentralización y privatización del sistema de salud chileno". Centro de Estudios Públicos, Revista de políticas públicas, No 39. Disponible en: <http://www.cepchile.cl/1_1640/doc/ descentralizacion_y_privatizacion_del_sistema_de_salud_chileno.html\#.U7m84ZR5ORK> [Consulta: 3 agosto 2014].

15 El D.L. No 2.763, Diario Oficial, 3 de agosto de 1979, reorganizó el Ministerio de Salud (MINSAL), creó los Servicios de Salud, el Fondo Nacional de Salud (FONASA), para que realizara las funciones financieras y de distribución presupuestaria; la Central de Abastecimiento (CENABAST), con el rol de abastecer de fármacos e insumos clínicos al sector; y el Instituto de Salud Pública (ISP), sucesor legal del Instituto Bacteriológico de Chile, con el rol de laboratorio nacional y de referencia. A todos estos servicios públicos se les dio el carácter de entes funcionalmente descentralizados, con personalidad jurídica y patrimonio propio, sujetos en su funcionamiento a la supervigilancia del Ministerio de Salud, debiendo sujetarse a sus políticas, normas y planes generales en el ejercicio de sus actividades.

${ }^{16}$ El artículo 29 de la Ley No 18.575 Orgánica Constitucional de Bases Generales de la Administración del Estado cuyo texto refundido, coordinado y sistematizado se fijó por el Decreto con Fuerza de Ley
} 
ridad de las potestades referidas a la ejecución de las acciones de salud, dotándolos para estos efectos de personalidad jurídica pública propia, de un patrimonio propio y cierta autonomía respecto del poder central ${ }^{17}$. En esta estructura, los Hospitales eran una dependencia del Servicio de Salud respectivo, así como antes lo fueran del SNS, por lo que no contaban con personalidad jurídica ni patrimonio propio, ni con facultades propias para la gestión de sus recursos ${ }^{18}$.

Este sistema se mantuvo hasta el año 2005, cuando entró en vigor la Ley No 19.937 denominada "de Autoridad Sanitaria y Gestión"19, que modificó el D.L. No 2.763 de 1979 con la finalidad de reestructurar el sector público de salud y reasignar las funciones del sistema en las acciones de rectoría, regulación, fiscalización, financiamiento y prestación de servicios ${ }^{20}$. Posteriormente, el D.L. No 2.763/1979 modificado, fue refundido, coordinado y sistematizado conjuntamente con las Leyes No 18.933 y No 18.469, por el D.F.L. No 1 de 2005 del Ministerio de Salud, actualmente vigente.

En lo que nos interesa, bajo la Reforma, los Servicios de Salud mantuvieron la condición de servicios públicos funcionalmente descentralizados, con personalidad jurídica, patrimonio propio y sujetos a la supervigilancia del Ministerio de Salud (artículo 16 incisos $2^{\circ}$ y $3^{\circ}$ D.F.L. No $1 / 2005$ ), pero dejaron de estar a cargo de la ejecución de acciones asistenciales y de fiscalización sanitaria, para cumplir una función de articulación, gestión y desarrollo de la Red Asistencial (artículo 16 inciso $1^{\circ}$ D.F.L. No 1/2005). Así las cosas, pasaron a ser los establecimientos integrantes de la Red Asistencial ${ }^{21}$, y no los Servicios de Salud, los entes ejecutores de las acciones de

No 1-19.653, de 2000, del Ministerio Secretaría General de la Presidencia, Diario Oficial, 17 de noviembre de 2001, señala: "Los servicios públicos serán centralizados o descentralizados. Los servicios centralizados actuarán bajo la personalidad jurídica y con los bienes y recursos del Fisco y estarán sometidos a la dependencia del Presidente de la República, a través del Ministerio correspondiente. Los servicios descentralizados actuarán con la personalidad jurídica y el patrimonio propios que la ley les asigne y estarán sometidos a la supervigilancia del Presidente de la República a través del Ministerio respectivo. La descentralización podrá ser funcional o territorial'.

17 Aylwin Azócar, Patricio y Azócar Brunner, Eduardo (1996). Derecho Administrativo. Santiago: Universidad Nacional Andrés Bello, p. 154.

${ }^{18}$ Véase el Capítulo IV “De los Hospitales”, Título I-VI- artículos 76-215, del Reglamento Orgánico de los Servicios de Salud, contenido en el derogado Decreto Supremo No 42 de 1986, Diario Oficial, 9 de diciembre de 1986.

19 En cuanto a la materia en examen, la Ley No 19.937 entró en vigencia el 1 enero de 2005, conforme establece su artículo decimo octavo transitorio.

${ }^{20}$ La Ley No 19.937 entrega nuevas facultades regulatorias al MINSAL, redistribuye las funciones regulatorias de los Servicios de Salud traspasándolas a las Secretarías Regionales Ministeriales (SEREMIS), reorganiza el MINSAL en dos Subsecretarías (de Salud Pública y Redes Asistenciales), crea la Superintendencia de Salud, establece un nuevo rol para los Servicios de Salud y crea la figura de los Establecimientos de Menor Complejidad y Autogestionados en Red.

21 "La Red asistencial de cada Servicio de Salud está constituida por el conjunto de establecimientos asistenciales públicos que forman parte del Servicio, los establecimientos municipales de atención primaria de salud de su territorio y los demás establecimientos públicos o privados que suscriban convenio con el Servicio de Salud respectivo (...)", artículo 17 D.F.L. No 1/2005 MINSAL. 
salud, las cuales, empero, deben ser controladas, organizadas y planificadas desde el propio Servicio en su calidad de "Gestor de Red" (artículo 21 D.F.L. No 1/2005). Bajo esta premisa, se buscó dotar a los hospitales con mayor complejidad técnica, desarrollo de especialidades, organización administrativa y número de prestaciones, de una mayor independencia en materias de gestión administrativa y presupuestaria, creándose la categoría de "Hospitales Autogestionados en Red"22 como establecimientos de salud dependientes y funcionalmente desconcentrados del correspondiente Servicio de Salud (artículos 31 incisos $1^{\circ}$ y $5^{\circ}$ D.F.L. No 1/2005) ${ }^{23}$.

Hay que precisar, para delimitar los efectos temporales de esta nueva regulación, que, conforme estableció el artículo 25A de la Ley No 19.937, para adquirir la categoría de Autogestión en Red, los hospitales debían acreditar el cumplimiento de una serie de requisitos y que dicha calidad se otorgaría mediante una resolución conjunta de los Ministerios de Salud y Hacienda. Sin perjuicio de ello, por disposición del artículo 15 transitorio de la misma ley, los 56 hospitales que allí se indican, que no hubiesen obtenido la mencionada calificación al 31 de enero de $2010^{24}$, pasarían a tener la calidad de Autogestionados a contar de esa fecha por el solo ministerio de la ley ${ }^{25}$.

Ahora bien, se podría afirmar que, siendo los Hospitales Autogestionados organismos dependientes y funcionalmente desconcentrados de los respectivos Servicios

\footnotetext{
22 "El concepto de autogestión implica dependencia de los hospitales en materia de la cartera de servicios (oferta sanitaria que es definida por la red pública), y una mayor desconcentración e independencia en materias de gestión, todo esto en un contexto de redes de atención estructuradas en torno a un Modelo de Atención Integral en Salud, el cual fue definido por el Ministerio de Salud como el conjunto de acciones organizadas del sistema, orientadas a promover la calidad de vida de las personas, consideradas en su integralidad tanto individual como social, y que se caracteriza por estar centrado en el usuario; por el énfasis en lo promocional y preventivo; por su enfoque familiar y comunitario y por garantizar la integralidad y continuidad de los cuidados junto a promover la resolución ambulatoria y participativa de las necesidades de salud". Artaza Barrios, Osvaldo (2008). "Los desafíos de la autogestión hospitalaria”. Revista Chilena de Pediatría, Vol. 79, No 2, abril, pp. 127-130. Disponible en: <http://www.scielo.cl/scielo.php?script=sci_ar ttext\&pid=S0370-41062008000200001> [Consulta: 3 agosto 2014].

${ }^{23}$ Los Establecimientos de Autogestión en Red se encuentran actualmente regulados en el Capítulo II, Título IV, Párrafos 1-4, artículos 31-44, del D.F.L. No 1/2005 MINSAL, y en el Capítulo II, Títulos I-VIII, artículos 10-35, del D.S. No 38 de 2005 de MINSAL, Diario Oficial, 29 de diciembre de 2005, que establece el Reglamento Orgánico de los Establecimientos de Salud de Menor Complejidad y de Establecimientos de Autogestión en Red. Supletoriamente se les aplican las disposiciones del Reglamento Orgánico de los Servicios de Salud, contenido en el D.S. No 140 de 2004 de MINSAL, Diario Oficial, 21 de abril de 2005.

${ }^{24}$ Artículo modificado por Ley No 20.319, Diario Oficial, 31 de diciembre de 2008. Originalmente, la Ley No 19.937 consideraba como fecha tope el 1 de enero de 2009.

25 Obtuvieron la calidad de Establecimientos de Autogestión en Red antes del 31 de enero de 2010 los siguientes 11 hospitales: en el año 2006, el Instituto Nacional del Cáncer (comuna de Independencia), el Hospital Dr. Luis Calvo Mackenna (comuna de Providencia) y el Hospital de Temuco; en el año 2007 los Hospitales Dr. Luis Tisné Brousse (comuna de Peñalolén), Instituto Nacional del Tórax (comuna de Providencia) y el Instituto Traumatológico Dr. Teodoro Gebauer (comuna de Santiago); en el año 2008, el Hospital Herminda Martín de Chillán, el Hospital San Juan de Dios de Los Andes, el Instituto Psiquiátrico Dr. José Horwitz Barak (comuna de Recoleta), el Hospital El Pino (comuna de San Bernardo) y el Hospital Exequiel González Cortés (comuna de San Miguel).
} 
de Salud y, por tanto, careciendo de personalidad jurídica propia, los Servicios de Salud debiesen responder jurídica, administrativa y patrimonialmente por aquellos. Sin embargo, el modelo de desconcentración administrativa que previno la Reforma para los Hospitales Autogestionados es un tanto más compleja y relativiza este planteamiento.

\subsection{Caracteristicas de la desconcentración funcional de los Hospitales Autogestionados}

Regulada en el artículo 33 inciso $1^{\text {o }}$ y final LOCBGAE ${ }^{26}$, la desconcentración funcional es una técnica de reparto de potestades administrativas que opera entre órganos que integran una misma persona jurídica pública -que puede ser centralizada o descentralizada- y que consiste en la transferencia permanente por ley de competencias de un órgano superior a otro inferior de la misma entidad, que actúan en el mismo ámbito territorial y cuyo objetivo es mejorar la gestión del servicio público sin alterar la línea jerárquica de dependencia entre el órgano superior y el inferior "desconcentrado" 27 .

Resulta característico de la desconcentración administrativa que, a consecuencia de la titularidad de la potestad transferida, al órgano desconcentrado se le atribuya la exclusiva responsabilidad por los actos ejecutados en ejercicio de dicha potestad, sin quedar subordinado en esta materia al control jerárquico de sus superiores. Sin embargo, esta responsabilidad no cambia el grado de imputación jurídica de la actuación administrativa, en la medida que tanto el órgano superior como el desconcentrado forman parte de la misma persona jurídica. Al contrario, en la técnica de descentralización administrativa, como se crea una persona jurídica independiente, el órgano descentralizado es titular de la potestad, de la responsabilidad consiguiente por su ejercicio y además, de la imputación de las consecuencias jurídicas de aquélla ${ }^{28}$.

Este último rasgo referido a la responsabilidad final por los actos ejecutados y a la imputación jurídica de ésta, es determinante en el análisis, dado que, si entendemos que el Hospital Autogestionado es un órgano funcionalmente desconcentrado del Servicio de Salud en los términos referidos anteriormente, al no contar con personalidad jurídica ni patrimonio propio - características propias de un ente descentralizado-debiese actuar con la personalidad jurídica y el patrimonio del Servicio y, por ende, a este último debiesen imputarse jurídicamente las consecuencias de

\footnotetext{
${ }^{26}$ El artículo 33 de la Ley No 18.575, señala: "Sin perjuicio de su dependencia jerárquica general, la ley podrá desconcentrar, territorial y funcionalmente, a determinados órganos" (inciso $1^{\circ}$ ). "La desconcentración funcional se realizará mediante la radicación por ley de atribuciones en determinados órganos del respectivo servicio" (inciso final).

27 Ferrada Bórquez (1999), pp. 120-123.

${ }^{28}$ Ferrada Bórquez (1999), pp. 120-123.
} 
los actos ejecutados por aquél en ejercicio de sus atribuciones -como por ejemplo, de aquellos constitutivos de una falta de servicio en la prestación asistencial-.

Sin embargo, la Ley de Autoridad Sanitaria al crear los Hospitales Autogestionados, a objeto de consolidar un modelo de gestión de servicio público especializado, que fortaleciera la autonomía y responsabilidad de estos, introdujo tres variaciones a la tradicional forma de constitución de un servicio funcionalmente desconcentrado: Primero, les delegó la representación judicial y extrajudicial del Servicio de Salud para el ejercicio de las atribuciones radicadas por ley en su esfera de competencia, fundamentalmente referidas a la ejecución de programas y acciones de salud, celebración de actos y contratos y la administración del personal destinado al establecimiento, con la sola obligación de que, notificada una demanda, deben ponerla en conocimiento del Director del Servicio de Salud en el plazo de 48 horas, para que éste adopte las medidas administrativas que procedieran, pudiendo intervenir como coadyuvante en cualquier estado del juicio (artículo 36 inciso final D.F.L. No 1/2005). Corrobora esta idea de dotar al Hospital de mayor autonomía, la norma del artículo 35 D.F.L. No 1/2005 que establece un deber de no injerencia al señalar: "La administración superior y control del Establecimiento [Autogestionado] corresponderán al Director. El Director del Servicio de Salud no podrá interferir en el ejercicio de las atribuciones que le confiere este Titulo al Director del Establecimiento [Autogestionado], ni alterar sus decisiones. Con todo, podrá solicitar al Director del Establecimiento la información necesaria para el cabal ejercicio de las funciones de éste".

Segundo, la ley los dotó de un patrimonio de afectación, preciso en cuanto a los fondos y bienes específicos que lo componen y al logro del fin concreto al que debe servir, de manera que en el ejercicio de sus atribuciones transferidas no comprometen sino los recursos y bienes afectos al cumplimiento de sus fines propios (artículo 31 inciso $6^{\circ}$ en relación a los artículos 42 y 43 D.F.L. No 1/2005).

Finalmente, la ley les asignó un presupuesto propio distinto del aprobado por la Ley de Presupuestos para el Servicio de Salud, aprobado por el Subsecretario de Redes Asistenciales, sobre la base del presupuesto aprobado por la Ley de Presupuestos al Servicio de Salud correspondiente. Luego, durante la ejecución del presupuesto, el Director del Hospital Autogestionado puede modificar el presupuesto propio del hospital y los montos determinados en sus glosas, haciendo los ajustes que estime necesarios; sin embargo, tales modificaciones pueden ser rechazadas por el Subsecretario de Redes Asistenciales, de acuerdo con las instrucciones impartidas por la Dirección de Presupuestos ${ }^{29}$ (artículo 36 letras d) y e) D.F.L. No 1/2005).

Es posible apreciar que partiendo de la base que tanto Servicio de Salud como Hospital forman parte de la misma persona jurídico-administrativa (Servicio de Salud), la especial desconcentración administrativa prevista por la Reforma dota

29 Sobre las Instrucciones para la ejecución de la Ley de Presupuestos del Sector Público 2014, véase: Dirección de Presupuesto. Disponible en: <http://www.dipres.gob.cl/594/w3-propertyvalue-15891.html> [Consulta: 3 agosto 2014]. 
a los Hospitales Autogestionados de una organización propia para gestionar independientemente el conjunto de competencias, el recurso humano y los medios materiales adscritos al servicio público, constituyéndolos en un centro de imputación de relaciones jurídicas pero sin llegar a personificarlos, sin crear una "autonomía funcional”, rasgos conceptuales que se confunden con aquellos distintivos de los entes descentralizados ${ }^{30}$.

\section{La legitimación pasiva de los Hospitales Autogestionados}

El modelo de gestión hospitalaria seguido por la Ley de Autoridad Sanitaria que dispuso a los Hospitales Autogestionados integrando la persona jurídica del Servicio de Salud, pero dotándolos a la vez de personalidad jurídica delegada para el ejercicio de sus atribuciones, de un presupuesto y un patrimonio de afectación destinado al cumplimiento de sus fines propios, nos permite deducir que son los Hospitales Autogestionados quienes detentan la legitimación pasiva en las acciones de responsabilidad por falta de servicio así como la titularidad jurídica para responder patrimonialmente por las indemnizaciones compensatorias de perjuicios.

Por cierto y relacionado con los especiales rasgos de la desconcentración administrativa de los Hospitales Autogestionados, y en línea con las contemporáneas doctrinas procesales ${ }^{31}$, este modelo también altera los criterios tradicionales de atribución de legitimación procesal de los entes públicos, que han entendido la capacidad de un órgano público para ser parte en un proceso jurisdiccional y actuar válidamente en él como demandante o demandado, dependiendo de si tiene o no personalidad jurídica, criterio vinculado a la categorización que distingue entre administración centralizada o descentralizada, donde en el primer caso, la capacidad para ser parte radicará en el Fisco y en el segundo, en el órgano por sí mismo, tal como se desprende de lo dispuesto en los artículos 29, 35 y 36 LOCBGAE.

En lo concerniente a la legitimación pasiva, esto es, la determinación de "frente a quién" ha de ser interpuesta una petición de tutela jurisdiccional para que ésta resulte subjetivamente eficaz, la utilidad de la distinción antes indicada radica en poder identificar al órgano público que está obligado frente al derecho que se hace valer mediante la pretensión procesal interpuesta. Sólo ese órgano entonces, puede ser considerado como un "demandado legítimo"32.

\footnotetext{
${ }^{30}$ Los Hospitales Autogestionados comparten varias de las características de las "Administraciones Institucionales" españolas, a excepción de la personalidad jurídica. Sobre la Administración Institucional véase García de Enterría Eduardo y Fernández Tomas-Ramón (2004). Curso de Derecho Administrativo I. Madrid: Civitas Ediciones, 12a edición, pp. 368-427.

${ }^{31}$ GutiérRez Silva, José Ramón (2009). "El presupuesto procesal de la capacidad de las personas jurídicas, en especial las de derecho público". Revista Chilena de Derecho, Vol. 36, No2, agosto, pp. 245-279. Disponible en: <http://dx.doi.org/10.4067/S0718-34372009000200003> [Consulta: 3 agosto 2014]; GonZÁLEZ Pillado, Esther (2004). "Comentarios prácticos a la Ley de Enjuiciamiento Civil”. Indret Procesal, Revista para el Análisis del Derecho, No 3, pp. 01-30.

32 "Ávila Aguilera c/Fernández Mora" (apelación sentencia cese pensión de alimentos): Corte de Apelaciones de Concepción, 29 de octubre de 2009, Rol F-392-2009.
} 
Sin embargo, tratándose de los Hospitales Autogestionados, este criterio no es aplicable porque estamos frente a entes que no tienen formalmente personalidad jurídica propia al ser órganos desconcentrados del Servicio de Salud pero, con todo, desde un punto de vista procesal, tienen plena capacidad para ser parte, tanto para solicitar la tutela de sus intereses (legitimación activa) cuanto para discutir u oponerse a las pretensiones hechas valer en su contra (legitimación pasiva).

La legitimación procesal de los Hospitales Autogestionados se infiere del artículo $25 \mathrm{D}$ inciso final de la Ley de Autoridad Sanitaria y actual artículo 36 inciso final del D.F.L. No 1/2005 MINSAL, que prescribe "Para todos los efectos legales, la representación judicial y extrajudicial del Servicio de Salud respectivo se entenderá delegada en el Director del Establecimiento [Autogestionado], cuando ejerza las atribuciones señaladas en este artículo (...)".

Esta norma, por una razón de conveniencia vinculada al propósito de hacer responsable al Hospital por las atribuciones competenciales transferidas por ley, define la aptitud del órgano que cuenta con la capacidad para ser parte directamente en juicio -el Hospital Autogestionado y la personería de quien ejecutará válidamente a su nombre los actos procesales- el Director del Hospital Autogestionado -sin necesidad de recurrir a la personalidad jurídica del Servicio de Salud-, que es lo que correspondería hacer de aplicarse los criterios tradicionales de atribución de legitimación procesal de los entes públicos en razón de su personalidad jurídica.

Pero además, desde un punto de vista práctico, no hay necesidad de recurrir a dicha personalidad jurídica tampoco para la ejecución de una eventual sentencia condenatoria de indemnización de perjuicios, puesto que acá lo que interesa es recurrir al patrimonio que responderá por dicha indemnización y no a la personalidad jurídica; y tratándose de los Hospitales Autogestionados, estos tienen un patrimonio propio de afectación, cuyos fondos y bienes están comprometidos explícitamente al cumplimiento de sus fines propios, esto es, a la ejecución de programas y acciones de salud así como a la compensación por daños a terceros causados a raíz de ello ${ }^{33}$.

${ }_{33}$ Para ilustrar este punto hay que considerar el principio de legalidad tanto del acto administrativo que ordena el pago de la indemnización de perjuicios como la legalidad presupuestaria para financiar dicho pago. Por ejemplo, el artículo 36, letra i), inciso $1^{\circ}$, del D.F.L. No 1/2005 MINSAL, previene, en lo que interesa que los Directores de los Establecimientos de Autogestión en Red, entre otras atribuciones, tienen la de "transigir respecto de derechos, acciones y obligaciones, sean contractuales o extracontractuales", añadiendo su inciso $2^{\circ}$ que "Los contratos de transacción deberán ser aprobados por resolución del Ministerio de Hacienda, cuando se trate de sumas superiores a cinco mil unidades de fomento" (Cfr. Dictamen No 74.157 de 2012 de Contraloría General de la República). En relación a esto, si bien el clasificador presupuestario (Decreto No 854/2004 de Hacienda) prevé que el gasto que demanda tanto este tipo de transacciones como el de una sentencia condenatoria ejecutoriada, debe imputarse al presupuesto vigente del organismo al Subtítulo 26 -Otros gastos corrientes- Ítem 02 -Compensaciones por daños a terceros y/o a la propiedad(esto es gastos por concepto de pagos como indemnizaciones por daños a las personas o a las propiedades causados por organismos del sector público. Pueden ser pagos para el cumplimiento de las sentencias que se encuentren ejecutoriadas, incluidas las de origen previsional, dictadas por autoridades competentes de acuerdo con el artículo 752 del Código de Procedimiento Civil u otras disposiciones legales, como pagos de gracia acordados extrajudicialmente), los presupuestos de los Hospitales Autogestionados suelen no 
Respecto de este punto, el Ministerio de Salud, mediante Circular No 1, de 29 de febrero de 2008, impartió expresas instrucciones a los Servicios de Salud y Hospitales Autogestionados en materia de representación judicial de los Establecimientos de Autogestión en Red, indicando que "la representación judicial del Establecimiento [Autogestionado], corresponde por ley a su Director, al que compete asumir -en su caso- las correspondientes acciones para su defensa, y que en el caso de acciones judiciales dirigidas contra el Establecimiento [Autogestionado], el resultado de las mismas solamente compromete los bienes y recursos del mismo, no implicando, en ningún caso, los bienes del respectivo Servicio de Salud. Sin perjuicio por cierto, que el Director del Servicio pueda, si lo estima conveniente, intervenir como tercero coadyuvante en dichos juicios" (el destacado es nuestro ${ }^{34}$.

\subsection{Tratamiento jurisprudencial de la legitimación pasiva de los Hospitales y Servicios Públicos de Salud}

Como se ha señalado, la legitimación pasiva de los Hospitales Autogestionados y/o de los Servicios de Salud va estrechamente ligada a la forma de organización administrativa prevista por la regulación de dichos servicios públicos y a las mayores o menores atribuciones que en materia de representación judicial en cada caso se les haya entregado.

Para determinar entonces, cuál de estos servicios públicos tiene la legitimación pasiva en el caso concreto de responsabilidad sanitaria institucional y en el entendido que las disposiciones de derecho público rigen in actum, es decir, regulan todas las situaciones comprendidas en el ámbito de sus normas desde la fecha en que entran en vigencia, salvo que prevean una fecha especial de vigencia o contengan preceptos en contrario ${ }^{35}$,debemos atender al momento de ocurrencia de los hechos constitutivos de la eventual falta de servicio del organismo para determinar la normativa aplicable y las potestades entregadas al órgano público comprometido. Así las cosas, y tal como señalamos al referirnos a los efectos temporales de esta nueva regulación, según dispuso la Ley de Autoridad Sanitaria en sus artículos 25A en relación con el 15 transitorio, los Hospitales Autogestionados pasaron a detentar la legitimación pasiva en vez de los Servicios de Salud, a partir de dos momentos:

tener liberado este ítem de gastos. Entonces, en la práctica, de ser condenado el Hospital al pago de una indemnización de perjuicios, debe solicitar al Servicio de Salud respectivo el financiamiento de este gasto, para lo cual éste, a su vez, debe requerir las respectivas modificaciones presupuestarias, las cuales pueden contemplar, por ejemplo, la redistribución de los Subtítulos 22 y/o incrementos de Ingresos Operacionales del Subtítulo 7 del presupuesto del Hospital para que proceda a financiar directamente el mencionado gasto con cargo a esa cuenta.

${ }^{34}$ Circular No 1, de 29 de febrero de 2008, del Ministerio DE SALUd. "Imparte instrucciones en materia de representación judicial de los Establecimientos de Autogestión en Red”. Disponible en <http://juridico1. minsal.cl/CIRCULAR_1_08\%20\%20Autogestionados.doc>.

${ }^{35}$ Cfr. Dictámenes de Contraloría General de la República Nos. 1.754, de 2010; 33.078, de 2010;19.788, de 2007 y 61.615, de 2008, entre otros. 
la fecha prevista en la resolución conjunta de los Ministerios de Salud y Hacienda, a contar de la cual se otorga la categoría de Autogestión en Red a aquellos establecimientos que se acreditaron voluntariamente; o bien, respecto de aquellos que no hubiesen obtenido la mencionada calificación, a contar del 31 de enero de 2010, fecha en que por el solo ministerio de la ley obtuvieron dicha calidad.

En el presente apartado se revisarán tres sentencias de apelación, de los años 2009, 2010 y 2014, que permitirán ilustrar la manera en que se ha abordado el tema de la legitimación pasiva de los Hospitales y Servicios de Salud en materia de responsabilidad sanitaria.

\subsection{1. "Astete Ortiz cl Hospital Luis Calvo Mackenna"36}

Se trata de una demanda de responsabilidad extracontractual por falta de servicio, a causa del fallecimiento de una menor de edad ocurrido el día 5 de noviembre de 1998, interpuesta por la madre de la menor en contra del Hospital Dr. Luis Calvo Mackenna y del Fisco de Chile, por estimar la demandante que el hospital era un establecimiento dependiente del Servicio de Salud Metropolitano Oriente (en adelante el Servicio Oriente) y, este último, un servicio perteneciente a la administración centralizada del Estado de Chile.

Por sentencia de 13 de marzo de 2006, el 27o Juzgado Civil de Santiago acogió la demanda en contra de ambos demandados, condenándolos a pagar en forma solidaria la suma de $\$ 250.000 .000$ por indemnización de perjuicios a título de daño moral. La Corte de Apelaciones de Santiago, en el año 2009, revocó el fallo, rechazando la demanda en todas sus partes y resolviendo que el Hospital Dr. Luis Calvo Mackenna carecía de legitimación pasiva en la acción ejercida en su contra. Posteriormente, la Corte Suprema, conociendo de los recursos de casación en forma y fondo interpuesto por la demandante, confirmó la sentencia impugnada, reiterando los argumentos expuestos por la Corte de Apelaciones de Santiago.

La doctrina del fallo en materia de legitimación pasiva está recogida en los considerandos 1) a 5) de la sentenciade la Corte de Apelaciones. Fundada en los artículos 16 y 18 del D.L. No 2.763 de 1979 y los artículos 29 y 36 de la LOCBGAE, la Corte precisa que los Servicios de Salud (en este caso, el Servicio Oriente) son organismos estatales, funcionalmente descentralizados, dotados de personalidad jurídica y patrimonio propio para la realización de las acciones de salud; que están a cargo de un Director, jefe superior del Servicio, que tiene su representación judicial y extrajudicial; y que no forman parte de la Administración Central del Estado. Señala que "la implementación de un sistema de descentralización en los servicios de salud, persiguió precisamente [que esas personas jurídicas] prestaran servicios con autonomía del Poder Central, y para lo cual, se les dotó de personalidady

36 "Astete Ortiz cl Hospital Luis Calvo Mackenna" (indemnización de perjuicios): 27o Juzgado Civil de Santiago, 13 de marzo de 2006, Rol No 1733-2000; Corte Apelaciones de Santiago, 7 de agosto de 2009, Rol No 3648-2006; Corte Suprema, 9 de diciembre de 2011, Rol No 7623-2009. 
patrimonio propio y autonomía operacional' (considerando $4^{\circ}$ ). Por las consideraciones referidas, la Corte resolvió acoger la alegación de falta de legitimación pasiva promovida por los demandados, estimando que no resultaba procedente accionar válidamente contra el Hospital ni contra el Fisco comprometiendo su patrimonio. En contra del Hospital, por carecer de personalidad jurídica y patrimonio propio, ya que depende del Servicio de Salud, y respecto del Fisco, por no corresponderle la representación judicial o extrajudicial del Servicio ya que este último no es un ente centralizado (considerando $5^{\circ}$ ).

La solución que adopta el fallo de la Corte es correcta en cuanto a la aplicación y a la interpretación que hace de la normativa orgánica del sector salud vigente a la fecha de ocurrencia de los hechos - 5 de noviembre de 1998-. Ello, porque tal como se indicó en apartados anteriores, el D.L. No 2.763, de 1979, estructuraba el sector público de salud en torno al Sistema Nacional de Servicios de Salud (SNSS), compuesto por Servicios de Salud descentralizados, a cargo de la ejecución directa de acciones de salud, integrados por Hospitales como unidades internas de su estructura organizacional que carecían de personalidad jurídica, patrimonio propio y facultades para la gestión propia de sus recursos. Sin embargo, en razón de lo señalado y considerando que esta normativa se encuentra modificada a contar del año 2005, no resulta procedente invocarla en la actualidad para referirse a la legitimación pasiva de los Hospitales y Servicios de Salud, como lo hace la doctrina más reciente en esta materia, ya que esta jurisprudencia resulta desactualizada en relación a la Reforma del sector salud.

\subsection{2. "Sánchez Candia cl Hospital Luis Calvo Mackenna"37}

Se trata de una demanda de responsabilidad extracontractual por falta de servicio y por hecho ajeno del artículo 2320 inciso $1^{\circ}$ del Código Civil, a causa del fallecimiento de una menor de edad, sucedido el día 18 de enero de 2006, dirigida en contra del Hospital Dr. Luis Calvo Mackenna, representado legalmente por el Director del Servicio de Salud Metropolitano Oriente (en adelante el Servicio Oriente).

Por sentencia de 29 de abril de 2009, el 120 Juzgado Civil de Santiago acogió la demanda interpuesta en contra del Hospital. Posteriormente, conociendo del recurso de apelación, la Corte de Apelaciones de Santiago revocó el fallo, rechazando la demanda en todas sus partes y resolviendo en definitiva, que no existía responsabilidad del Servicio Oriente, persona jurídica de quien dependía el Hospital Calvo Mackenna ${ }^{38}$, ni por falta de servicio ni por el hecho de sus dependientes.

\footnotetext{
37 "Sánchez Candia cl Hospital Luis Calvo Mackenna" (indemnización de perjuicios): 12 Juzgado Civil de Santiago, 29 de abril de 2009, Rol No 16763-2006; Corte Apelaciones de Santiago, 29 de mayo de 2010, Rol No 4046-2009.

${ }^{38}$ La Corte de Apelaciones resolvió que no existía responsabilidad del Servicio de Salud Metropolitano Oriente ni por falta de servicio ni por el hecho de sus dependientes, puesto que estimó que la muerte de
} 
La doctrina en materia de legitimación pasiva está recogida en el considerando $1^{\circ}$ del fallo de la Corte, el cual, sin hacer referencia a norma legal alguna, manifiesta, al igual que en el caso anterior, que el Hospital Calvo Mackenna carece de personalidad jurídica y depende administrativamente del Servicio de Salud Oriente, entidad que sí es una persona jurídica. Ahora bien, sin perjuicio de ello y a diferencia del caso anterior, la Corte subsanó el vicio de falta de legitimación pasiva, considerando que, aunque la demanda estaba dirigida en contra del Hospital Calvo Mackenna, representado por el Director del Servicio, fue el Servicio Oriente quien la contestó y figuró como parte demandada durante todo el juicio en el entendido que se afectaban sus intereses, de manera que debía entenderse que la acción se dirigió, en realidad, en contra del Servicio Oriente (considerando 1\%). En Astete c/ Hosp. Calvo Mackenna, sin embargo, la Corte rechazó la demanda debido a que ésta no fue dirigida y ni siquiera notificada válidamente al Servicio de Salud en la persona de su Director, lo que incluso fue representado oportunamente por la Directora del Hospital sin que se hubiera reparado la situación (considerando $5^{\circ}$ ).

El razonamiento del presente fallo, a pesar de no invocar expresamente normativa alguna, es correcto al afirmar que al momento de los hechos -18 de enero de 2006-, el Hospital Calvo Mackenna carecía de personalidad jurídica y dependía administrativamente del Servicio Oriente, persona jurídica que detentaba la representación del mencionado Hospital.

Con todo, resulta necesario precisar que a la fecha de ocurrencia de los hechos, nos encontrábamos con la Reforma de salud en marcha, ya que desde el año anterior regía el D.L. No 2.763, de 1979, modificado por la Ley de Autoridad Sanitaria y Gestión, que creaba la institución de los Hospitales Autogestionados con las características que hemos ya visto; pero a dicha época el Hospital Calvo Mackenna aún no adquiría la calidad de Autogestionado, categoría que adquirió mediante el proceso de evaluación previsto en el artículo 25A de la Ley No 19.937, por Resolución Exenta No 367, de 2006, de los Ministerios de Salud y Hacienda ${ }^{39}$.

Por lo anterior, a la fecha de ocurrencia de los hechos la situación del Hospital era idéntica a la prevista en el fallo anterior, por lo que tampoco resulta adecuado invocar en la actualidad esta jurisprudencia para explicar la legitimación pasiva de los Hospitales Autogestionados.

la menor se debió a las múltiples y graves enfermedades de que padecía y no a que el servicio no hubiese funcionado o hubiese funcionado deficiente o tardíamente. Asimismo, descartó la acción de responsabilidad civil por hecho ajeno porque la demanda no se dirigió en contra de ninguna persona natural y nunca se demostró en el proceso la culpa de algún miembro del Hospital que hubiese conducido a la muerte de la niña.

${ }^{39}$ Resolución Exenta No367 de 2006 de los Ministerios de Salud y Hacienda, Diario Oficial, 11 de marzo de 2006. Disponible en: <http://juridico1.minsal.cl/RESOLUCION_367_06.doc> [Consulta: 3 agosto 2014]. 


\subsection{3. "Contreras Vásquez c/ Hospital Barros Luco Trudeau”ło}

Se trata de una demanda de responsabilidad extracontractual por falta de servicio, interpuesta en contra del Hospital Barros Luco Trudeau, a causa del fallecimiento de una mujer ocurrido el día 23 de junio de 2008. Por sentencia de 30 de octubre de 2013, el 2o Juzgado Civil de San Miguel acogió la demanda deducida por las hijas de la mujer fallecida, en contra del Hospital Barros Luco. El 22 de enero de 2014, la Corte de Apelaciones de San Miguel confirmó la sentencia apelada con declaración de elevar el monto de la indemnización a pagar por el Hospital en su calidad de "órgano autogestionado de la Administración del Estado". Esta sentencia fue acordada con un voto en contra, que estuvo por revocar el fallo en alzada y rechazar la demanda, al estimar que el Hospital Barros Luco carecía de legitimación pasiva en la acción ejercida en su contra.

Es difícil sostener que este fallo sienta alguna doctrina relevante pertinente a la materia en examen puesto que los argumentos de que se vale para afirmar la legitimación pasiva del Hospital Barros Luco, así como los del voto de la disidencia para negarla, se fundan en interpretaciones erradas de la normativa vigente.

La Corte razona al igual que el juez de primera instancia, entendiendo que el Hospital Barros Luco es un "órgano autogestionado de la Administración del Estado”. No señala otro fundamento, pero suponemos que se refiere a la calidad de Establecimiento Autogestionado en Red que invocó el Hospital, representado por su Director, al momento de contestar la demanda. Sin embargo, resulta que, a la fecha de ocurrencia de los hechos -23 de junio de 2008-, el Hospital Barros Luco aún no era Autogestionado, ya que no adquirió dicha calidad sino hasta el 31 de enero de 2010 por el solo ministerio de la ley, de lo cual resulta que, aunque el Hospital haya sido finalmente emplazado en juicio en el año 2012, oportunidad en que ya era un Establecimiento Autogestionado, no procedía acoger acción alguna en su contra fundada en hechos acaecidos con anterioridad a que adquiriera dicha categoría, porque, como se ha venido señalando, al haber sido un organismo dependiente y subordinado del Servicio de Salud, a este último le correspondía la responsabilidad por los actos constitutivos de la falta de servicio y la imputación de las consecuencias jurídicas de aquéllos y no al Hospital.

El voto disidente, por su parte, se pronunció por rechazar la demanda fundando la falta de legitimación pasiva del Hospital Barros Luco en el criterio tradicional de atribución de capacidad de ser parte en atención a la personificación del ente público que ya revisamos en párrafos precedentes. Señala que "fue demandado y condenado el Hospital Barros Luco, pese a que es un hecho público y notorio que dicho establecimiento de salud forma parte del Servicio de Salud Metropolitano Sur

40 “Contreras Vásquez c/ Hospital Barros Luco Trudeau” (indemnización de perjuicios): 2o Juzgado Civil de San Miguel, 30 de octubre de 2013, Rol No 75882-2011; Corte Apelaciones de San Miguel, 22 de enero de 2014, Rol No 2292-2013. 
(...) de manera que no cuenta con personalidad juridica propia (...). De acuerdo al artículo 16 del Decreto con Fuerza de Ley No 1 del año 2005, del Ministerio de Salud, que creó el Servicio de Salud Metropolitano Sur, entre otros, dispone que los Servicios de Salud son organismos estatales, funcionalmente descentralizados, dotados de personalidad jurídica y patrimonio propio. Por lo anterior el Hospital Barros Luco Trudeau no es una persona juridica de derecho público y, en consecuencia no es capaz de ejercer derechos y contraer obligaciones ni de ser representado judicial y extrajudicialmente, como si lo es el Servicio de Salud Metropolitano Sur al que pertenece, persona jurídica cuya representación le corresponde a su Director como jefe superior del servicio (...) Que, en consecuencia, al carecer de personalidad jurídica, el Hospital Barros Luco Trudeau no tiene capacidad legal para ser emplazado, y por ende carece de legitimación pasiva y no es posible actuar en su representación, sin que pueda entenderse emplazado el Servicio de Salud Metropolitano Sur (...)" (considerandos $2^{\circ}$ y $3^{\circ}$ del voto disidente).

$\mathrm{Al}$ respecto, podemos efectuar las siguientes observaciones:

1. El argumento de la disidencia sería correcto siempre que se refiriera a la condición jurídico-administrativa del hospital con anterioridad al 31 de enero de 2010, pero no queda claro que este criterio cronológico de análisis fuera previsto al momento de resolver. Sobre todo si consideramos que el voto de minoría se sustenta en el artículo 16 del D.F.L. No 1/2005 del Ministerio de Salud, referido al rol de "Gestor de Red" de los Servicios de Salud, norma que por las razones que examinamos anteriormente, debe entenderse en el contexto de una Red Asistencial integrada por Hospitales Autogestionados, debiendo, entonces, haberse considerado en el análisis además las normas regulatorias de los Autogestionados, especialmente las previstas en la Ley No 19.937 referidas al reconocimiento de dicha calidad.

2. Si supusiéramos que se razonó en este sentido, o sea, que el Hospital Barros Luco al momento de ocurrencia de los hechos aun no adquiría la calidad de Autogestionado, resulta dudoso afirmar como un hecho público y notorio que el mencionado Hospital por carecer de personalidad jurídica propia carece también de legitimación pasiva. Para arribar a esta conclusión era indispensable, primeramente, despejar la condición jurídico-administrativa del hospital al momento de ocurrencia de los hechos -órgano dependiente del Servicio o autogestionado- y recién ahí, pasar a analizar si dicha condición le confería o no legitimación pasiva en el proceso.

3. Como se ha indicado, es incorrecto afirmar que un organismo público por el hecho de carecer de personalidad jurídica no es capaz de ejercer derechos y contraer obligaciones ni de ser representado judicial y extrajudicialmente. Como se expresó con anterioridad, esto depende de la regulación en concreto que la legislación efectúe de las competencias de dicho organismo.

Por las razones expuestas, el voto de minoría no debía rechazar la demanda fundando la falta de legitimación pasiva del Hospital Barros Luco en el argumento 
tradicional de ser un ente sin personalidad jurídica, condición que se mantiene tanto antes como después de adquirir la categoría de Autogestionado, sino en la circunstancia que, a la fecha de ocurrencia de los hechos, este Hospital dependía del Servicio de Salud Metropolitano Sur bajo la misma estructura que la establecida desde los años 80, por lo que la capacidad procesal invocada por el Hospital durante su defensa carecía de sustento legal, significaba actuar con abuso de poder, sin tener la autoridad conferida por ley y fuera de la órbita de su competencia ya que esta correspondía, conforme a derecho, al Servicio de Salud, vulnerando la prohibición constitucional que contempla el inciso $2^{\circ}$ del artículo $7^{\circ}$ de la Constitución Política ${ }^{41}$.

\section{CONCLUSIONES}

Hemos podido constatar que, desde la entrada en vigencia en el año 2005 de la Ley No 19.937 de Autoridad Sanitaria y Gestión, son los propios Hospitales Autogestionados y no los Servicios de Salud -puntualmente, unos desde el año 2006 y otros desde el 2010- quienes detentan la legitimación pasiva en las acciones de responsabilidad por falta de servicio y la titularidad jurídica para responder patrimonialmente por las indemnizaciones compensatorias de perjuicios.

$\mathrm{Al}$ atribuirles una organización funcionalmente desconcentrada con las particularidades que revisamos, la relación que existe entre Hospital Autogestionado y Servicio de Salud más que propiamente de dependencia es una relación de dirección estratégica dentro del ámbito de gestión del servicio público, lo que refleja que los Hospitales Autogestionados son entes instrumentales a los fines de los Servicios de Salud en cuanto a la gestión técnica de la provisión de servicios públicos de salud. Sin embargo, al tener una mayor capacidad de autodeterminación y de autogestión en la esfera de sus competencias, traducida en una autonomía ejecutiva u operativa así como financiera, los Hospitales Autogestionados se constituyen en centros de imputación de relaciones y posiciones jurídicas, al ser titulares propios de esas relaciones y posiciones, no obstante formar parte de la misma persona jurídico-administrativa del Servicio de Salud, superando así los criterios tradicionales de atribución de legitimación procesal de los entes públicos en razón de su personalidad jurídica: Los Hospitales Autogestionados tienen capacidad para ser parte en juicio y responden patrimonialmente por sus actos propios, no obstante, por definición, carezcan de personalidad jurídica y patrimonio propio.

La doctrina más reciente al tratar la responsabilidad sanitaria institucional y, puntualmente el tema de la legitimación pasiva de los servicios públicos de salud,

41 "Ruiz Matamala c/I. Municipalidad de Pedro Aguirre Cerda" (apelación sentencia nulidad derecho público): Corte de Apelaciones de San Miguel, 11 de julio de 2014, Rol 228-2014. 
no ha incorporado estos conceptos y ha sostenido erradamente que por los Hospitales Autogestionados responden jurídica y patrimonialmente los Servicios de Salud. Como hemos visto, para ello se ha basado en jurisprudencia desactualizada en relación al estado actual de la Reforma de la Salud, básicamente, al no aplicar la normativa prevista en la Ley No 19.937 de Autoridad Sanitaria y Gestión y no prestar especial atención al momento de ocurrencia de los hechos constitutivos de la falta de servicio y al momento en que el Hospital comprometido adquirió la categoría de Establecimiento Autogestionado en Red.

\section{BiBLIOGRAFÍA CITADA}

Artaza Barrios, Osvaldo (2008). "Los desafíos de la autogestión hospitalaria”. Revista Chilena de Pediatría, Vol. 79, No 2, abril, pp. 127-130. Disponible en: <http://www. scielo.cl/scielo.php?script=sci_arttext\&pid=S0370-41062008000200001> [Consulta: 3 agosto 2014].

Aylwin Azócar, Patricio y Azócar Brunner, Eduardo (1996). Derecho Administrativo. Santiago: Universidad Nacional Andrés Bello.

Cárdenas Villarreal, Hugo y Moreno Molinet Jaime (2011). Responsabilidad Médica. Estándares jurisprudenciales de la falta de servicio. Santiago: LegalPublishing Thomson Reuters, $1^{\text {a }}$ edición, 285 pp.

Ferrada Bórquez, Juan Carlos (1999). "El Estado Administrador en Chile: de unitario centralizado a descentralizado y desconcentrado". Revista de Derecho (Valdivia), Vol. $\mathrm{X}$, diciembre, pp. 115-123. Disponible en: <http://mingaonline.uach.cl/scielo. php?pid=S0718-09501999000200011\&script=sci_arttext> [Consulta: 3 agosto 2014].

García de Enterría Eduardo y Fernández Tomas-Ramón (2004). Curso de Derecho Administrativo I. Madrid: Civitas Ediciones, 12a edición.

Goic G., Alejandro y Armas M., Rodolfo (2003). "Descentralización en salud y educación: La experiencia chilena”. Revista Médica de Chile, Vol. 131, No 7, pp. 788-798. Disponible en: <http://dx.doi.org/10.4067/S003498872003000700012> [Consulta: 3 agosto 2014].

González Pillado, Esther (2004). "Comentarios prácticos a la Ley de Enjuiciamiento Civil”. Indret Procesal, Revista para el Análisis del Derecho, No 3, pp. 1-30.

GutiérRez Silva, José Ramón (2009). "El presupuesto procesal de la capacidad de las personas jurídicas, en especial las de derecho público". Revista Chilena de Derecho, Vol. 36, No 2, agosto, pp. 245-279. Disponible en:<http://dx.doi. org/10.4067/S0718-34372009000200003> [Consulta: 3 agosto 2014].

Inostroza, Manuel y Nancuante, Ulises (2013). "La experiencia chilena de administrar los Hospitales Autogestionados en Red 2005-2012: ¿Un instrumento consolidado y definitivo o una transición a un nuevo tipo de prestador público, para una nueva etapa de la Reforma de Salud". 95 propuestas para un Chile

Revista de Derecho - Escuela de Postgrado No 6, diciembre 2014 
mejor, Estudio de Trasfondo Grupo Res Publica Chile, Capítulo 15, pp. 121-181. Disponible en: <http://95propuestas.cl/site/wp-content/uploads/2013/05/ la-experiencia-chilena-de-administrar-los-hospitales-autogestionados-en-redmanuel-inostroza-y-ulises-nancuante.pdf> [Consulta: 3 agosto 2014].

Méndez, Claudio A., Miranda, Christian, Torres, M. Cristina y Márquez, Myriam (2012). "Implementación de la política de hospitales autogestionados en Chile: percepción de los profesionales hospitalarios". Revista Chilena de Salud Pública, Vol. 16, No 3, pp. 247-255. Disponible en: <http://www.revistasaludpublica.uchile.cl/index.php/RCSP/article/download/23158/24509> [consulta: 03 agosto 2014].

Méndez, Claudio A., Miranda, Christian, Torres, M. Cristina y Márquez, Myriam (2013). "Política de autogestión hospitalaria en Chile: percepciones de los tomadores de decisiones". Revista Panameña de Salud Pública, Vol. 33, No 1, pp. 47-53. Disponible en: <http://www.captura.uchile.cl/handle/2250/133406> [Consulta: 3 agosto 2014].

Miranda R., Ernesto (1990). "Descentralización y privatización del sistema de salud chileno". Centro de Estudios Públicos, Revista de politicas públicas, No 39. Disponible en: <http://www.cepchile.cl/1_1640/doc/descentralizacion_y_privatizacion_del_sistema_de_salud_chileno.html\#.U7m84ZR5ORK> [Consulta: 3 agosto 2014].

Nancuante Almonacid, Ulises y Romero Celedón, Andrés (2008). La reforma de la salud. Instituto de Políticas Públicas y Gestión, Salud y Futuro-UNAB, Santiago: Editorial Biblioteca Americana, 1a edición, 570 pp.

Nancuante Almonacid, Ulises, Romero Celedón, Andrés y Sotomayor Klapp, Roberto (2012). Régimen jurídico de la salud. Santiago: LegalPublishing Thomson Reuters, $1^{\text {a }}$ edición, 707 pp.

Titelman, Daniel (2000). "Reformas al Sistema de salud en Chile: Desafíos pendientes". CEPAL-Serie Financiamiento del Desarrollo, Unidad de Financiamiento para el Desarrollo, División de Comercio Internacional y Financiamiento para el Desarrollo, Naciones Unidas, Vol. 104, septiembre. Disponible en: <www. cepal.org/publicaciones/xml/0/5640/lcl1425e.pdf> [Consulta: 3 agosto 2014].

Tocornal CoOper, Josefina (2010). "Responsabilidad civil por infecciones intrahospitalarias". Revista Chilena de Derecho, Vol. 37, No 3, diciembre, pp. 477-504. Disponible en: <http://dx.doi.org/10.4067/S0718-34372010000300004> [Consulta: 3 agosto 2014].

Tocornal CoOper, Josefina (2014). La Responsabilidad Civil de Clinicas y Hospitales. Santiago: LegalPublishing-Thomson Reuters, $1^{\text {a }}$ edición, 375 pp.

Vergara, Marcos (2007). "Reforma del sector de la salud en Chile: Avances y problemas en la implementación”. Serie En Foco No 112. Disponible en: <http:// www.expansiva.cl/publicaciones/en_foco/detalle.tpl?iddocumento $=0407200$ 7114141>[Consulta: 3 agosto 2014]. 


\section{NORMAS CITADAS}

Constitución Política de la República.

Ley No 10.383, modifica la Ley No 4.054 relacionada con el Seguro Obligatorio, Diario Oficial, 8 de agosto de 1952.

Ley No 18.575, Orgánica Constitucional de Bases Generales de la Administración del Estado cuyo texto refundido, coordinado y sistematizado se fijó por el Decreto con Fuerza de Ley No 1-19.653, de 2000, del Ministerio Secretaría General de la Presidencia, Diario Oficial, 17 de noviembre de 2001.

Ley No 19.937, modifica el D.L. No 2.763 de 1979 con la finalidad de establecer una nueva concepción de la autoridad sanitaria, distintas modalidades de gestión y fortalecer la participación ciudadana, Diario Oficial, 24 de febrero de 2004.

Ley No 20.319, modifica la Ley No 19.937, impidiendo que los establecimientos de salud no calificados como de autogestión en red al 1 de enero de 2009, pasen a tener dicha calidad por el solo ministerio de la ley, Diario Oficial, 31 de diciembre de 2008.

D.L. No 2763, Diario Oficial, 3 de agosto de 1979.

D.F.L. No 1 de 2005, del Ministerio de Salud, fija texto refundido, coordinado y sistematizado del D.L. No 2763 de 1979 y de las leyes No 18.933 y 18.469, Diario Oficial, 24 de abril de 2006.

Decreto No 854/2004, del Ministerio de Hacienda, determina clasificaciones presupuestarias, Diario Oficial, 2 de diciembre de 2004.

Decreto No 42 de 1986, del Ministerio de Salud, Reglamento Orgánico de los Servicios de Salud, Diario Oficial, 9 de diciembre de 1986.

Decreto No 140 de 2004, del Ministerio de Salud, Reglamento Orgánico de los Servicios de Salud, Diario Oficial, 21 de abril de 2005.

Decreto No 38 de 2005, del Ministerio de Salud, Reglamento Orgánico de los Establecimientos de Salud de Menor Complejidad y de Establecimientos de Autogestión en Red, Diario Oficial, 29 de diciembre de 2005.

\section{JURISPRUDENCIA CITADA}

"Astete Ortiz cl Hospital Luis Calvo Mackenna" (indemnización de perjuicios): 270 Juzgado Civil de Santiago, 13 de marzo de 2006, Rol No 1733-2000; Corte Apelaciones de Santiago, 7 de agosto de 2009, Rol No 3648-2006; Corte Suprema, 9 de diciembre de 2011, Rol No 7623-2009.

"Ávila Aguilera c/Fernández Mora" (apelación sentencia cese pensión de alimentos): Corte de Apelaciones de Concepción, 29 de octubre de 2009, Rol F-392-2009.

"Contreras Vásquez cl Hospital Barros Luco Trudeau" (indemnización de perjuicios): 2o Juzgado Civil de San Miguel, 30 de octubre de 2013, Rol 
No 75882-2011; Corte Apelaciones de San Miguel, 22 de enero de 2014, Rol No 2292-2013.

"Ruiz Matamala c/I. Municipalidad de Pedro Aguirre Cerda" (apelación sentencia nulidad derecho público): Corte de Apelaciones de San Miguel, 11 de julio de 2014, Rol 228-2014.

"Sánchez Candia c/Hospital Luis Calvo Mackenna" (indemnización de perjuicios): 12º Juzgado Civil de Santiago, 29 de abril de 2009, Rol No 16763-2006; Corte Apelaciones de Santiago, 29 de mayo de 2010, Rol No 4046-2009. 